\title{
Consumption and Effect of Artificial Sweeteners and Artificially Sweetened Products on Lebanese Population
}

\author{
Marwa Al Mousawi ${ }^{1 b}$, Sami Tlais ${ }^{2 a b *}$, Ali Alkatib ${ }^{1 a}$, Hassan S. Hajj Hussein ${ }^{2 b}$
}

\author{
${ }^{1}$ Nutrition and Food Science Department, Lebanese International University, Beirut ${ }^{\mathrm{a}} /$ Rayak $^{\mathrm{b}}$, Lebanon. \\ ${ }^{2}$ Biological and Chemical Department, Lebanese International University, Bekaa ${ }^{\mathrm{a}} /$ Rayak $^{\mathrm{b}}$, Lebanon. \\ *Correspondence should be addressed to: Sami Tlais, Email: sami.tlais@liu.edu.lb
}

\begin{abstract}
Non-caloric or non-nutritive artificial sweeteners are chemically synthesized or extracted from natural sources to replace regular sugar. The main reasons behind using artificial sweeteners are weight loss, blood sugar control for diabetic people, and dental caries prevention. Although many studies support the use of sweeteners and artificially sweetened products, others focus on the long-term negative consequences of their usage. A survey was conducted to explore the effect and prevalence of non-caloric tabletop sweeteners and non-caloric sweetened products consumption among Lebanese population based on their Body Mass Index (BMI), sex, age, and health. The survey was completed by a sample of 457 subjects. The questionnaire included consumption pattern, brands used, and the frequency of consuming artificial sweeteners and sweetened products. Data showed that $30 \%$ of Lebanese population consumes artificial sweeteners as tabletop while $31 \%$ consumes artificially sweetened products. Sucralose was the most consumed sweetener; however, its daily intake range was far below its Acceptable Daily Intake (ADI). The highest percentage of consumers $(41 \%)$ made the shift to sweeteners from regular tabletop sugar around one year prior to this study. With increasing age, there was a decrease in the percentage of respondents who observed "weight loss" due to replacing regular sugar with sweeteners. The study determined that the majority of respondents (66\%) were unaware of the side effects of these sweeteners. Also, the majority of diabetic respondents (78\%) and (57\%) did not observe any decrease in HbAlc and blood sugar levels, respectively. In a complementary survey that targeted Lebanese dietitians, $78 \%$ recommended the use of tabletop sweeteners instead of tabletop sugar, while $52 \%$ did not support the consumption of artificially sweetened products. Also, the survey showed that "Nevella" was the most recommended brand by (57\%) of dietitians.
\end{abstract}

Keywords-Lebanon, sweetener, effect, artificial, population, dietitians.

\section{INTRODUCTION}

Human's affection for sweet-tasting food is inborn. Studies have shown preference for sweet-tasting products in newborns and infants [1]. Several studies confirm a tight correlation between the consumption of sucrose over a lifetime and tooth decay, as well as other diseases such as obesity, diabetes, and cancer[2,3]. Obesity is a major problem throughout the world and could lead to other severe diseases such as type-2 diabetes and cardiovascular heart diseases. Rising rates of obesity require avoiding the over consumption of calories and searching for non-caloric alternatives to sugar [4].This has created an increased demand for innovative low-calorie foods; and as a result, non-nutritive sweeteners have become very popular [5]. ISSN: 2456-1878

https://dx.doi.org/10.22161/ijeab.54.6
"Sodium saccharin" is the oldest synthetic sweetener which was discovered in 1879, followed by "cyclamate" in 1937 [6]. Cyclamate was originally approved for commercial use by the U.S. Food and Drug Administration (FDA) in 1951, then after 18 years it was banned by the same agency[7]. These innovative sweeteners, which mimic the taste of sugar, can be thousands offolds sweeter than sugar (sucrose), and that it is why they are called "intense sweeteners"[8]. They are either "artificial sweeteners", which are chemically synthesized or "natural sweeteners", which are extracted from natural origin (Table 1). Until now, there were many concerns and debates regarding their safety; In 2014, a Nature study showed that saccharin directly modulates the composition and function of 
microbiome and induces dysbiosis accounting for the downstream glucose intolerance phenotype in the mammalian host [9]. Accumulating evidence suggests that frequent consumers of these sugar substitutes may be at increased risk of excessive weight gain, metabolic syndrome, type 2 diabetes, and cardiovascular disease [10]. Although many studies demonstrated the hazardous effects of the long-term consumption of artificial sweeteners, there are still others in the medical and health communitythat defend them[11]. In 2017, a Lebanese study showed that the brand "Canderel ${ }^{\mathbb{R} "}$ was the only NNS (non-nutritive sweetener) that caused an increase in insulin levels without any effect on blood glucose in healthy subjects, which putthe consumer at risk of developing diabetes or prediabetes [12]. The largest US cohort study of postmenopausal women found a positive correlation between higher intake of ASB (artificial sweetened beverages) (twice or more daily) and the incidence of ischemic stroke, especially the small artery occlusion subtype, coronary heart disease (CHD), and all-cause mortality [13]. With the increasing potential risks of sweeteners, further studies on the effect of their consumption are needed.

In Lebanon, there is little or no data about the consumption of artificial sweeteners (AS) or artificially sweetened products (ASP). The main objective of this study was to estimate the rate of consumption of artificial sweeteners and sweetened products among Lebanese population. The second objective was to highlight the dietitians' recommendations toward the consumption of AS. The data collected in this study argues if there is an emerging need to take urgent action to prevent the risk of overconsumption of such products and to collect additional data about the side effects of AS.

Table 1. Different sweeteners with their origin, corresponding ADI (Acceptable Daily Intake), sweetness intensity, calories they provide, and regulatory status [14].

\begin{tabular}{|c|c|c|c|c|c|}
\hline Sweetener & Origin & $\mathrm{ADI}^{\mathrm{a}}$ & Sweetness $^{\mathrm{b}}$ & $\overline{\mathrm{Kcal} / \mathrm{g}}$ & Regulatory Status \\
\hline $\begin{array}{c}\text { Acesulfame-K } \\
\text { (E950) }\end{array}$ & synthesized & 15 & $200 x$ & 0 & Approved (21 CFR 172.800) \\
\hline $\begin{array}{c}\text { Advantame } \\
\text { (E969) }\end{array}$ & synthesized & 32.8 & $20000 x$ & 0 & Approved (21 CFR 172.803) \\
\hline $\begin{array}{c}\text { Aspartame } \\
\text { (E951) }\end{array}$ & synthesized & 50 & $200 x$ & 4 & Approved (21 CFR 172.804) \\
\hline $\begin{array}{c}\text { Neotame } \\
\text { (E961) }\end{array}$ & synthesized & 0.3 & $4150 x^{c}$ & 0 & Approved (21 CFR 172.829) \\
\hline $\begin{array}{c}\text { Saccharin } \\
\text { (E954) }\end{array}$ & synthesized & 15 & $450 x^{c}$ & 0 & Approved (21 CFR 180.37) \\
\hline $\begin{array}{l}\text { Steviol } \\
(\text { E960) }\end{array}$ & natural & 4 & $300 x^{c}$ & 0 & GRAS $^{d}$ \\
\hline $\begin{array}{c}\text { Siraitia- } \\
\text { grosvenorii }\end{array}$ & natural & - & $175 x^{c}$ & 2 & GRAS d \\
\hline $\begin{array}{c}\text { Sucralose } \\
\text { (E955) }\end{array}$ & synthesized & 5 & $600 x$ & 0 & Approved (21 CFR 172.831) \\
\hline
\end{tabular}

${ }^{a} \mathrm{mg} / \mathrm{kg}$ bodyweight/day

${ }^{b}$ Multiplier of sweetness intensity compared to table sugar (Sucrose)

${ }^{c}$ Average

${ }^{d}$ Generally Recognized as Safe 


\section{METHODS}

\subsection{Study design, site and subjects}

A cross-sectional study design was applied from August 11, 2019 till September 13, 2019 and the sample size was 457 Lebanese respondent aged 18+ of both sexes (170 males and 287 females) from different regions of Lebanon. For the second survey, the sample size was 54 dietitians distributed all over Lebanon aged 21-39 years.

2.2 Data collection and analysis

A food frequency questionnaire was used to estimate the knowledge and consumption pattern of artificial sweeteners. Survey had 24 questions close ended with general information such demographic characteristics (sex and age), medical history, and consumption pattern of artificial sweeteners (AS) and artificially sweetened products (ASP). In addition, the questionnaire includes questions about weight, blood sugar, and HbA1c level change as observed by respondents. Respondents' anthropometric data namely height and weight were asked about in the questionnaire. Body mass index (BMI) was calculated by dividing the body weight in kilograms by the square of height in meters $\left(\mathrm{kg} / \mathrm{m}^{2}\right)$ to get four categories: underweight: $\mathrm{BMI}<18.5 \mathrm{~kg} / \mathrm{m}^{2}$, normal weight: BMI 18.5 $24.9 \mathrm{~kg} / \mathrm{m}^{2}$, overweight: BMI $25-29.9 \mathrm{~kg} / \mathrm{m}^{2}$, and obese: $\mathrm{BMI} \geq 30 \mathrm{~kg} / \mathrm{m}^{2}$. Dietitians' survey was conducted to get their opinion about AS consumption, products, and brands which they recommend. The questionnaires were sent to respondents via communicating applications.

\subsection{Statistical Analysis}

Data management was conducted using the Statistical Package for Social Science (SPSS) (IBM SPSS Statistics Version 23). For all analyses, P-value $<0.05$ was used to detect statistically significant difference. Data were analyzed using chi-squared test for categorical data.

\section{RESULTS AND DISCUSSION}

The percentage of consumption of tabletop AS among Lebanese population was almost similar to that of ASP as illustrated in Fig. 1A. A total of 457 respondents participated in this survey (170 males, 287 females), where $30 \%$ of respondents consumed AS (artificial sweeteners) as tabletop and $30 \%$ of respondents consumed ASP. The percentage obtained is very close to that observed in an Egyptian study that was done on Alexandria University students in 2017 where $31 \%$ of the respondents were consumers of tabletop artificial sweeteners [15].Likewise, these results matched a study aimed to assess the trends of artificial sweeteners consumption among young adults in the USA, and revealed that the prevalence of consumption of artificial sweeteners was up to $30 \%$ among the
American population in 2014 [16]. Our results show that only $20 \%$ of the whole sample was consumers of both tabletop and products artificially sweetened. Figure 1B shows the percentage of consumption duration. Where a high percentage of respondents $(41 \%)$ started using AS as early as one year or less prior to this study.
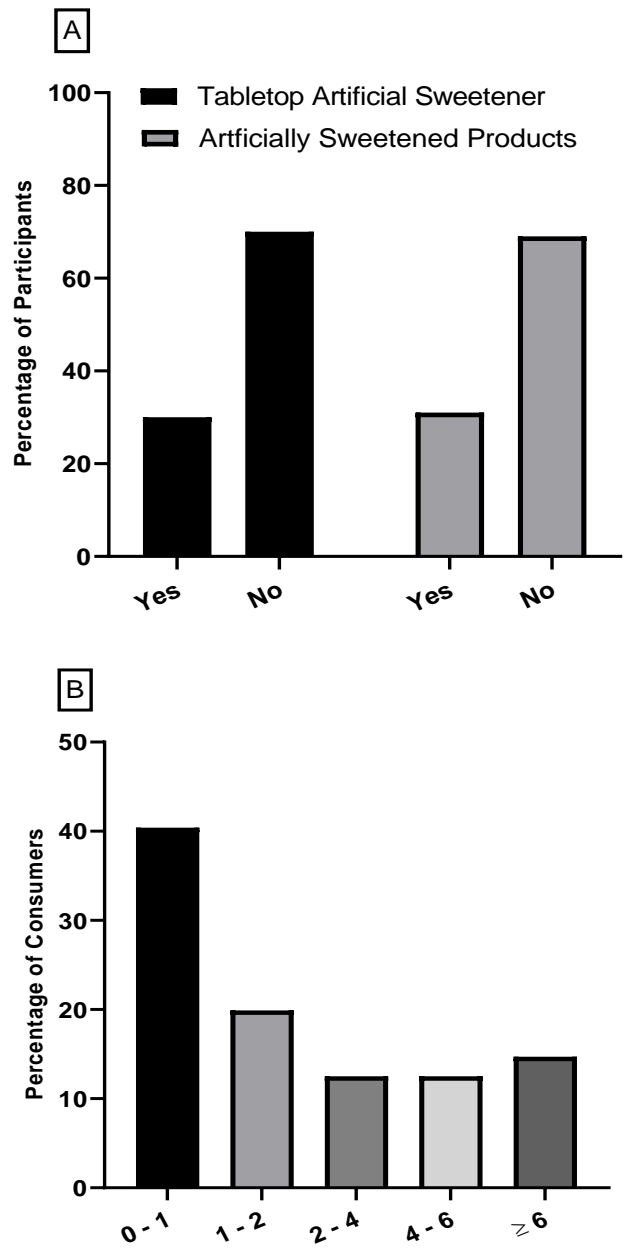

Fig.1. Percentage of participants using tabletop artificial sweeteners and artificially sweetened products (Fig. 1A).Percentage of AS consumers with different time intervals (years; Fig. 1B).

The percentages of male and female consumers are $28 \%$ and $31 \%$, respectively, and both significantly differ $(\mathrm{p}<0.0001)$ from non-consumers (Table 2). Data in this study show that the percentage of diabetic male consumers is $21 \%$ with no significant difference $(p>0.05)$ from diabetic female consumers which is $14 \%$. Highest percentage of consumers belong to the 36-45 years age group (42\%), whereas $48 \%$ of this age group reported that they suffered from diseases such as diabetes, cardiovascular heart disease, and blood pressure. Unlike the other age groups, the percentage of male consumers in the $36-45$ years is $(59 \%)$, which was significantly $(\mathrm{p}<0.05)$ 
higher than the female consumers (41\%).Respondents who were 18-20 years and 21-25 years of age showed the highest percentage of non-consumers $(81 \%$ and $75 \%$, respectively). This is related to their health condition where $91 \%$ of the $18-20$ age group and $96 \%$ of the $21-25$ age group reported that they did not suffer from any disease. These percentages differ from the Egyptian study where the highest percentage of consumers is of 20 to less than 22 years old $(35 \%)$ [15]. Statistical analysis shows that $59 \%$ of $18-20$ age group and $72 \%$ of $21-25$ age group of Lebanese none consumers had normal body weight.

Table 2 shows that $23 \%$ of consumers reported that they do not suffer from any disease, but $45 \%$ of them consumed tabletop AS to control their body weight and $21 \%$ to enjoy the sweet taste. The statistical data shows that there is a significant difference $(\mathrm{p}<0.0001)$ among BMI (Body Mass Index) categories between consumers and non-consumers, where $31 \%$ of overweight and $50 \%$ of obese respondents were consumers of tabletop sugar, but there was no significant difference in consumption of sweetened products among age groups, gender or BMI categories $(\mathrm{p}>0.05)$.

Although there is a big debate regarding the benefits and risks of AS, our study determined that $53 \%$ of consumers believe that AS are healthier than tabletop sugar, $21 \%$ had no idea whether it is a healthier choice or not, but $26 \%$ thought that it is not a healthy alternative to tabletop sugar and yet they still continued to consume it any way. Sixty nine percent of overweight respondents and $56 \%$ of obese respondents considered AS to be a healthier option compared to regular tabletop sugar.

With respect to the purpose behind AS usage, respondents gave a variety of answers. The majority of respondents (62\%)consumed AS to control their body weight, $25 \%$ to enjoy the sweet taste, $9 \%$ to keep blood sugar level low (diabetic respondents), and $4 \%$ to avoid dental caries. The highest percentage of those who consumed AS to control their body weight is in the 36-45 age group (82\%). One third of the respondents within 18-20 and 26-35 age groups consumed AS to enjoy the sweet taste, while "diabetes" was the main reason for AS intake for $42 \%$ of the respondents in the $46+$ age group.

Concerning the frequency of AS consumption, 29\% consume it once daily and only $9 \%$ consume it more than two times per day (Table 3), while the rest consume it less frequently. Table 3 shows that the highest percentage (76\%) of respondents consume 1 pill/sachet each time. With no significant difference among age groups ( $p>0.05)$ with respect to the number of pills consumed, the highest percentage (78\%) of 1 pill/sachet consumers was for 26-35 years age group. Lebanese population mainly consume AS in hot beverages such as coffee and tea with a percentage of $87 \%$. The percentage of males $(92 \%)$ who consume AS in hot beverages is significantly higher than females (84\%).

Table 2. Distribution of consumers of Tabletop artificial sweeteners among gender, age groups, BMI categories and diseases suffering from. (***: very highly significant)

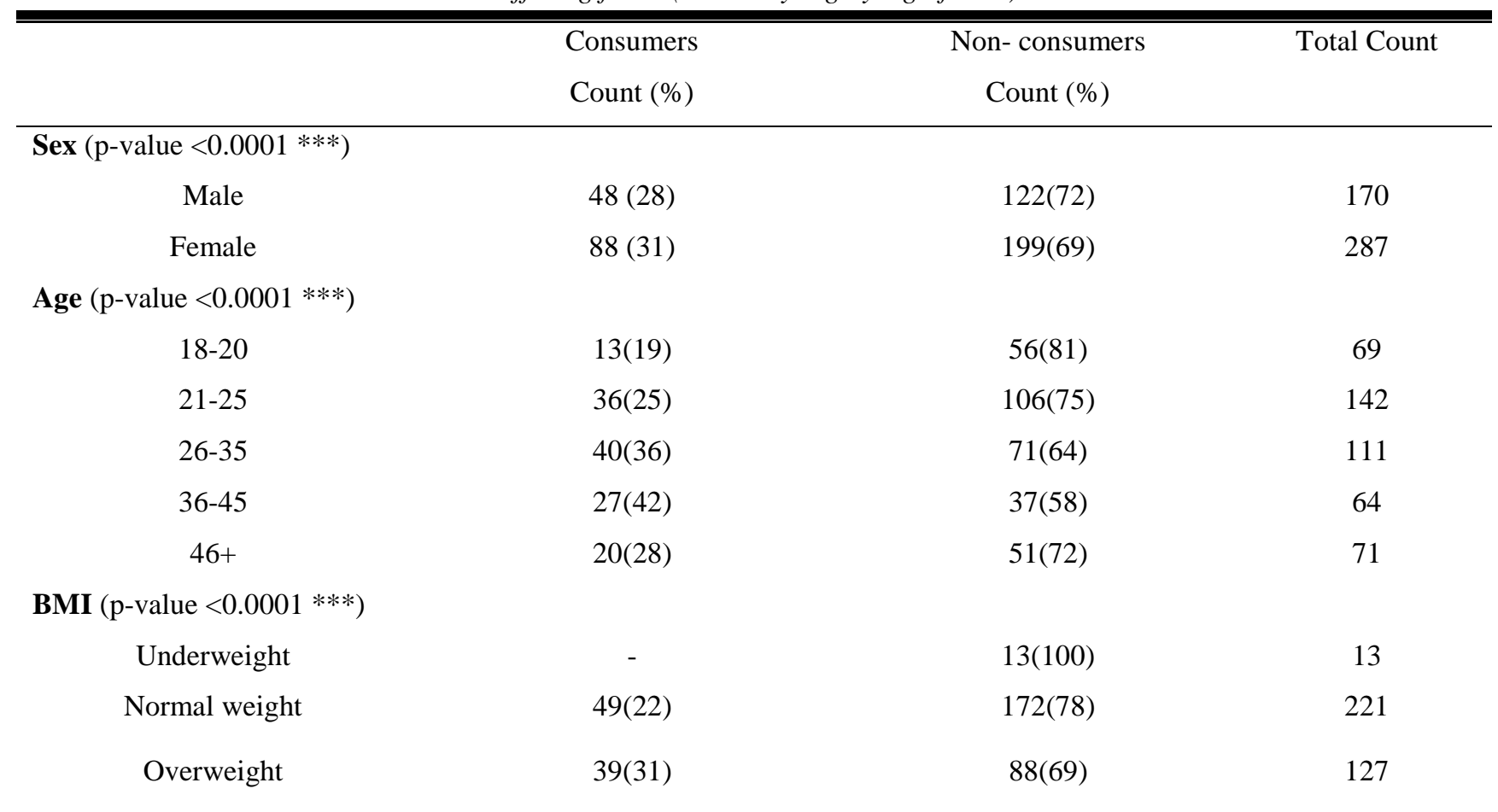


Obese

Disease (p-value $<0.0001 * * *)$

$\begin{array}{cc}\text { Obesity } & 28(67) \\ \text { Diabetes } & 22(71) \\ \text { Blood pressure } & 2(11) \\ \text { Other diseases } & 6(22) \\ \text { No disease } & 78(23)\end{array}$

$48(50)$

Different industrial and marketing companies have been investing in producing a substitute for sucrose. The most common brands of AS that are consumed among Lebanese population are: "Bloby", "Canderel", "Nevella", and
"Splenda" which have sucralose, while "Marinas Lixol" and "Sussli" have a mix of sodium cyclamate and sodium saccharin as the active ingredients.

Table 3. Frequency and amount of consumption of tabletop artificial sweeteners.

\begin{tabular}{ccccccc}
\hline \hline & $>$ 2times/day & 1 time/day & $\begin{array}{c}3-4 \text { times/ } \\
\text { week }\end{array}$ & $\begin{array}{c}2-3 \\
\text { times/week }\end{array}$ & $\begin{array}{c}2-3 \\
\text { times/month }\end{array}$ & Total \\
\hline $\begin{array}{c}1 \text { pill or } \\
\text { sachet/time }\end{array}$ & 5 & 32 & 16 & 20 & 30 & $103(76 \%)$ \\
$\begin{array}{c}2-3 \text { pills or } \\
\text { sachets /time } \\
>3 \text { pills or }\end{array}$ & 6 & 6 & 4 & 6 & 6 & $28(21 \%)$ \\
$\begin{array}{c}\text { sachets/time } \\
\text { Total }\end{array}$ & $13(9 \%)$ & $39(29 \%)$ & $20(15 \%)$ & $27(20 \%)$ & $37(27 \%)$ & $136(100 \%)$ \\
\hline \hline
\end{tabular}

A high percentage $(78 \%)$ of dietitians recommend the consumption of AS instead of regular sugar. This result was obtained through a separate survey that targeted Lebanese dietitians who are distributed over all Lebanese governorates. Fifty seven percent of dietitians recommended the brand "Nevella". The most probably reason for this recommendation could be the presence of probiotics in "Nevella" brand."Bloby" (sucralose) was the most consumed brand (25\%) among Lebanese population, followed by Canderel tablets (sucralose) (21\%) (Fig.2A). Results in this study are matched with the most consumed AS among Alexandria University students in Egypt which is sucralose [15]; moreover, sucralose was heavily consumed among overweight and obese Indians[11]. In this study, the intake amounts $(\mathrm{mg} / \mathrm{Kg})$ of sucralose, sodium saccharin and sodium cyclamate were much lower than the corresponding ADI of each sweetener. The mean daily intake of sucralose among the Lebanese population is $0.188 \mathrm{mg} / \mathrm{kg}$ with a range of $0.06-0.66 \mathrm{mg} / \mathrm{kg}$. The sucralose daily intake formost of respondents is between
0.06-0.21 $\mathrm{mg} / \mathrm{Kg}$ body weight/day, but all of these presented ranges are far below the ADI of sucralose which is stated by FDA as $5 \mathrm{mg} / \mathrm{kg}$ body weight (Fig. 2B). Similarly, sodium saccharin and sodium cyclamate intake amounts $(\mathrm{mg} / \mathrm{Kg})$ were below their ADI. The respondents mean daily intake of saccharin is $0.054 \mathrm{mg} / \mathrm{kg}$ and of cyclamate is $0.54 \mathrm{mg} / \mathrm{kg}$.

One of the main reasons for the consumption of AS is body weight control, $27 \%$ of consumers noticed weight loss when they consumed AS as a sugar substitute while $40 \%$ of consumers did not notice any weight change. The remaining $33 \%$ of consumers were not sure about their weight change. 

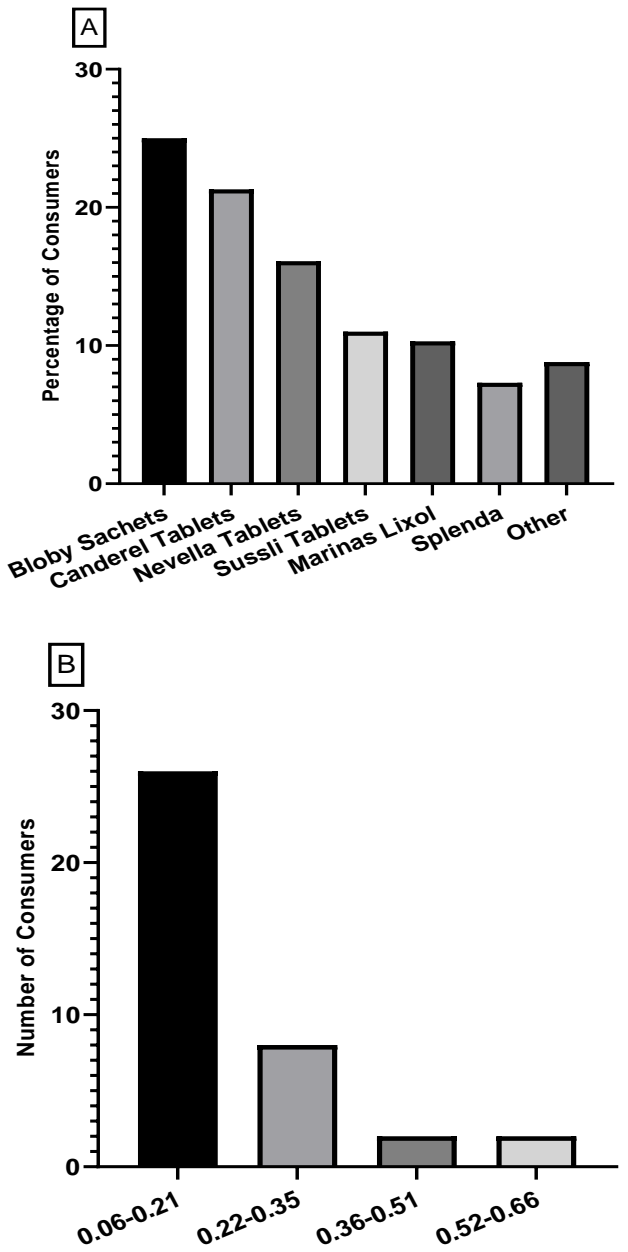

Fig.2. Percentage of most consumed tabletop artificial sweeteners by Lebanese population (Fig. 2A).Frequency of consumption of the four sucralose ranges $(\mathrm{mg} / \mathrm{kg}$ body weight/ day; Fig. 2B).

The percentages of obese and overweight respondents that observed weight decrease upon using AS are 38\% and 28\% respectively, with no significant difference $(\mathrm{p}>0.05)$ (Table 4).

Figure 3A shows the frequency of consumption of artificially sweetened products. Where $51 \%$ of ASP consumers consume it 2-3 times/month and only 9\% consume it on a daily basis. As well as, $52 \%$ of the lebanese dietitians do not recommend the consumption of AS products. Chocolate bars are the most consumed zerosugar products $(71 \%)$, followed by soft drinks $(65 \%)$, and chewing gums (59\%). Based on the responses, AS side effects are limited to stomach discomfort (10\%) and headache $(7 \%)$.
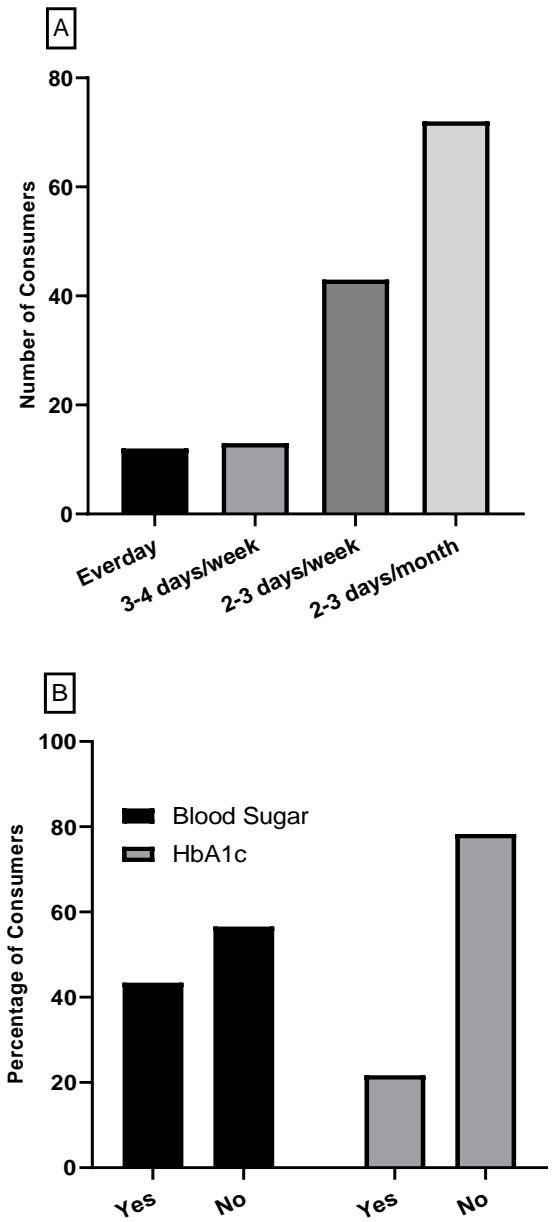

Fig.3.Frequency of consumption of artificially sweetened products (Fig. 3A).Answers of diabetic respondents about the decrease in blood sugar or HbAlc upon using artificial sweeteners (Fig. 3B).

As for the diabetic respondents, $57 \%$ and $78 \%$ did not observe a decrease in blood sugar and $\mathrm{HbA1c}$, respectively, upon the consumption of AS (Fig. $3 B$ ).However, studying the duration of usage of diabetic respondents reveals that 4 to 6 years interval consumers observed significantly higher blood sugar decrease compared to $2-<4$ years consumers. Whereas, less than one-year consumers did not observe any significant decrease in blood sugar. On the other hand, comparing the consumption duration of AS of HbAlcdid not show any significant differences.

Our data shows that the Lebanese population lack the knowledge of the side effects of AS consumption, where $20 \%$ of respondents linked AS intake to cancer and $66 \%$ did not state any possible side effects. The remaining $14 \%$ of consumers said that it causes problems in liver, kidney and heart. In comparison to the Indian study, only $7 \%$ of Indian respondents were able to state the later side effects [11]. 
Table 4. Respondents observations of weight decrease upon using artificial sweeteners with respect to sex, age and BMI categories.

\begin{tabular}{|c|c|c|c|}
\hline & No & Not sure & Yes \\
\hline & Count $(\%)$ & Count $(\%)$ & Count $(\%)$ \\
\hline \multicolumn{4}{|c|}{ Sex $(p$-value <0.303) } \\
\hline Male & $15(31)$ & $19(40)$ & $14(29)$ \\
\hline Female & $39(44)$ & $26(30)$ & $23(26)$ \\
\hline \multicolumn{4}{|c|}{ Age (p-value $<0.0001 * * *)$} \\
\hline $18-20$ & $5(38)$ & $4(31)$ & $4(31)$ \\
\hline $21-25$ & $15(42)$ & $5(14)$ & $16(44)$ \\
\hline $26-35$ & $22(55)$ & $8(20)$ & $10(25)$ \\
\hline $36-45$ & $8(30)$ & $14(52)$ & $5(19)$ \\
\hline $46+$ & $4(20)$ & $14(70)$ & $2(10)$ \\
\hline \multicolumn{4}{|c|}{ BMI (p-value <0.096) } \\
\hline Underweight & - & - & - \\
\hline Normal weight & $26(53)$ & $15(31)$ & $8(16)$ \\
\hline Overweight & $14(36)$ & $14(36)$ & $11(28)$ \\
\hline Obese & $14(29)$ & $16(33)$ & $18(38)$ \\
\hline
\end{tabular}

\section{CONCLUSION}

In conclusion, the present study highlights, for the first time, the percentage of Lebanese population that consumes artificial sweeteners and artificially sweetened products. The highest percentage $(42 \%)$ of consumers was determined to be within the 36-45 years age group. Even though a considerable high percentage of the population consumes AS, the mean intake was determined to be within the ADI (sucralose average intake was $0.188 \mathrm{mg} / \mathrm{kg}$ ) as set by the FDA (sucralose ADI is $5 \mathrm{mg} / \mathrm{kg}$ bw/ day). However, some of the Lebanese sweeteners have cyclamate which was banned by FDA but not by Joint FAO/WHO Expert Committee on Food Additives (JECFA).According to this study, AS new consumers are on the rise probably due to an increase in obesity awareness and an increase in the number of dietitians who recommend AS intake. However, it was alarming to discover that a large portion of the Lebanese population is still unaware of the side effects of AS.A large number of AS consumers (40\%) did not observe any decrease in body weight upon substituting tabletop sugar with AS. Also,the percentage of diabetics who did not observe a decrease in blood sugar and HbA1c was high. These results cast doubts on effectiveness of artificial sweeteners in controlling blood sugar levels. Further studies on the effect of AS and
AS products on the body weight, blood sugar, and HbA1c are needed.

\section{REFERENCES}

[1] Maone, T. R., Mattes, R. D., Bernbaum, J. C., \& Beauchamp, G. K. (1990). A new method for delivering a taste without fluids to preterm and term infants. Developmental Psychobiology, 23(2), 179-191. doi:10.1002

[2] Ludwig, D. S., Peterson, K. E., \& Gortmaker, S. L. (2001). Relation between consumption of sugar-sweetened drinks and childhood obesity: a prospective, observational analysis. The Lancit, 357(9255), 505-508. doi:10.1016

[3] Jiang, Y., Pan, Y., Rhea, P. R., Tan, L., et al, P. (2016). A Sucrose-Enriched Diet Promotes Tumorigenesis in Mammary Gland in Part through the 12-Lipoxygenase Pathway. Cancer Research, 76, 24-29.

[4] Kapadiya Dhartiben, B., \& Aparnathi, K. (2017). Chemistry and Use of Artificial Intense Sweeteners. International Journal of Current Microbiology and Applied Sciences, 6, 1283-1296. doi:10.20546

[5 ] Tandel, R. K., 2011. Sugar substitutes: Health controversy over perceived benefits.. Journal of Pharmacology and Pharmacotherapeutics, 2, 236-243.doi: 10.4103/0976500X.85936 
[6] Khan, R., \& Aroulmoji, V. (2018). Low Calorie HighIntensity Sweeteners. International Journal of Advanced Science and Engineering, 5, 934-947. doi:10.29294

[7] Weihrauch, M. R., \& Diehl, V. (2004). Artificial sweetenersdo they bear a carcinogenic risk? Annals of Oncology, 15, 1460-1465. doi:10.1093

[8] Chattopadhyay, S., Raychaudhuri, U., \& Chakraborty, R. (2011). Artificial sweeteners - a review. J Food Sci Technol, 51, 611-621. doi:10.1007

[9] Suez, J., Korem, T., Zeevi, D., Zilberman-Schapira, et al. Elinav, E. (2014). Artificial sweeteners induce glucose intolerance by altering the gut microbiota. Nature, 514, 181 197. doi:10.1038

[10] Swithers, S. E. (2013). Artificial sweeteners produce the counterintuitive effect of inducing metabolic derangements. Trends in Endocrinology and Metabolism, 24. doi:10.1016

[11] Bhagyasri, A., Naveen Kumar, R., Balakrishna, N., \& Sudershan Rao, V. (2016). Exposure Assessment of Artificial Sweeteners among Type 2 Diabetic, Overweight and Obese Individuals. The Indian Journal of Nutrition and Dietetics, 53, 268-276. doi:10.21048

[12] Imad, J., Wehbe, T., \& Abou Jaoude, E. (2017). A comparative study of three non-nutritive sweeteners effects on insulin and glucose in healthy, non-diabetic adults. Insights in Nutrition and Metabolism, 1(2), 73-79.

[13] Rahmani, Y. M., Kamensky, V., Mnson, J. E., et al. (2019). Artificially Sweetened Beverages and Stroke, Coronary Heart Disease, and All-Cause Mortality in the Women's Health Initiative. Stroke, 50, 555-562. doi:10.1161

[14] FDA. (2014). FDA. Retrieved april 13, 2020, from https://www.fda.gov/food/food-additives-

petitions/additional-information-about-high-intensitysweeteners-permitted-use-food-united-states

[15] Tayel , D. I., Khamis, N. A., \& Darwish, O. A. (2017). Artificial Sweeteners Consumption among Alexandria University Students, Egypt. Journal of High Institute of Public Health, 47(1), 1-7.

[16] Drewnowski, A., \& Rehm, C. D. (2014). Consumption of Low-Calorie Sweeteners among U.S. Adults Is Associated with Higher Healthy Eating Index (HEI 2005) Scores and More Physical Activity. Nutrients, 6, 4389-4403. doi: 10.3390 